\title{
Are label-free investigations the best approach to drug discovery?
}

“...new-generation label-free techniques have been implemented in various stages of drug discovery process including hit identification, lead optimization, target engagement determination, drug safety assessment and clinical diagnostics."

Keywords: antitarget $\bullet$ druggability $\bullet$ drug safety $\bullet$ hit identification $\bullet$ label-free binding technique $\bullet$ label-free cell phenotypic technique $\bullet$ lead selection $\bullet$ polypharmacology

Label-free techniques, in broad terms, are not new to drug discovery. Before the target-directed approaches took the center stage three decades ago, drugs were mostly discovered through phenotypic assessment of isolated tissues and whole animals without labels. With the rise of combinatorial chemistry, molecular biology, genomics and pharmacology in the past decades, labeling techniques have become dominating in early drug discovery process. Compared with traditional approaches, labeling techniques provide clear advantages in sensitivity, throughput and molecular specificity. The ability to perform high-throughput screening (HTS) with high sensitivity empowers labeling techniques to rapidly identify maximally selective lead molecules for many targets of interest. The molecular specificity permits the application of molecular and chemical knowledge to investigate specific molecular hypotheses. However, labeling techniques have caveats associated with label interference, cell engineering and target/mechanism of action constrain. Fluorescent and luminescent labels may alter the functions of probe molecules, proteins, or cells, thus introducing bias or artifacts in results $[1,2]$. The common use of engineered cells for screening precludes the investigation of drug action in native and physiologically relevant environments. The target/mechanism of action is predetermined in a given screen, but may be irrelevant to the disease pathogenesis, non-druggable, or inefficacious [3]. Furthermore, drug molecules identified may not have sufficient therapeutic index, due to its unknown but detrimental antitarget activity [4]. Recent analysis has shown that target-based approaches are more productive for discovering follow-on drugs, but less for first-in-class drugs, compared with phenotypic approaches [5]. Thus, there is high demand in implementing innovative technologies to discover drugs, in particular, for unmet therapeutic needs and unexploited biological mechanisms.

The past decade has witnessed the great expansion of label-free platforms and methods with enhanced and/or totally new capabilities. Compared with labeling approaches, certain label-free techniques (e.g., resonant waveguide grating, RWG) have comparable or even better sensitivity and throughput for cell-based screening, while others (e.g., mass spectroscopy [MS]) may have better molecular specificity even at the proteome level. Nowadays, new-generation label-free techniques have been implemented in various stages of drug discovery process including hit identification, lead optimization, target engagement determination, drug safety assessment and clinical diagnostics [6]. Of note, given that these techniques differ greatly in detection and operational principles and have their own advantages and caveats, the choice of techniques should be guided by the primary goals of specific drug discovery programs. Nonetheless, all label-free techniques do not need to use labels, reporters and engineered cells, whereas some (e.g., RWG) offer additional advantages associated with their ability to measure the kinetics in vitro and the holistic functional consequences in living cells of drug-target interactions.

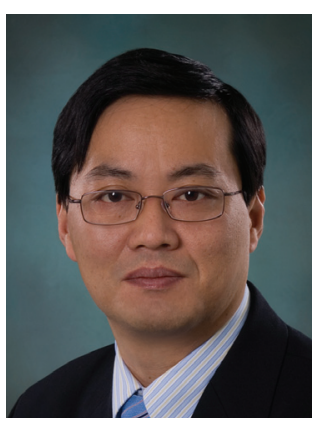

Ye Fang

Biochemical Technologies, Science \& Technology Division, Corning Incorporated, Corning, NY, USA fangy2@corning.com 
Label-free techniques have been used to determine almost every important aspect of drug-target interactions starting from affinity to kinetics, structure and thermodynamics, all of which are important to assess the druggability of compounds [7]. First, all drugs need to bind to their target(s) to exert effects; the binding affinity of a drug to its primary target is a traditional surrogate for its in vivo efficacy prediction. Label-free biosensors can measure the affinity of a drug binding to its target in different environments, which is essential to comprehend drug-target interactions in vivo where environments are diverse and complex. Surface plasmon resonance (SPR) and RWG can measure the affinity of drugs binding to purified receptors immobilized on the surface, while surface acoustic wave can measure the affinity of drugs binding to the receptor at the surface of living cells, and backscattering interferometry can determine drug-target binding affinity in homogenous solution or in vivo-like environment. Second, the kinetics, in particular, residence time (reciprocal of $\kappa_{\text {off }}$ ), of a drug is believed to be more critical to its clinical efficacy and safety profiles than binding affinity [8]. SPR is the standard technique to determine binding kinetics. RWG under microfluidics not only enables kinetic profiling with increased throughput, but also manifests the effect of binding kinetics on receptor signaling in living cells $[9,10]$. SPR imaging permits selectivity profiling of a specific drug against an array of targets, while biolayer interferometry is useful for kinetic profiling of biologic drugs with moderate throughput. Third, the structural features of drug binding are useful to determine druggability; that is, the likelihood of being able to modulate a target with a small molecule drug [11]. Structure-based techniques such as $\mathrm{x}$-ray, NMR and in silico docking can identify possible druggable sites of targets, enabling rational drug design. Fourth, the thermodynamic signatures including free energy, enthalpy and entropy are essential to medicinal chemistry optimization, and can be obtained using isothermal titration calorimetry.

\section{"...labeling techniques have caveats associated with label interference, cell engineering and target/mechanism of action constrain."}

Label-free binding techniques, in particular microplate-based RWG, have been used in HTS for hit identification. NMR spectroscopy is particularly valuable for validating hits and ligand binding, as well as discovering active fragments including weak binders without preknowledge of binding interactions [12]. Unlike labeled techniques that are often limited to detect compounds competing with the same binding site of the labeled probe used, label-free can detect hits for all possible binding sites of the target protein. With the ever-reducing cost of producing high-quality and purified proteins, label-free binding based on screening may become affordable in the near future.

Label-free cell phenotypic techniques (e.g., RWG and electric biosensor) have also been employed in HTS, and even ultra-HTS using native cells for hit identification. These techniques permit profiling and screening of drug molecules with a wide coverage in targets, pathways, cellular processes and cell types [13,14]. Owing to its noninvasive and holistic measure, labelfree technique allows for multistep screen campaigns that can simultaneously identify different classes of ligands for the target of interest (e.g., agonists, biased agonists, antagonists and allosteric modulators for a $\mathrm{G}$ protein-coupled receptor) with relatively low-false positives and negatives [6,15]. This is unlike conventional label-dependent screens which typically result in high-negative rates (mostly due to the bias defined by the molecular hypothesis for screening) and high-false positive rates (mostly related to the chemo-physical properties [e.g., aggregation, colors or fluorescence] of compounds, and the presence of compensatory targets/pathways) [14]. For label-free screens, hits can be selected from the library based on a desired size of response, or based on similarity of the cell phenotypic profiles of compounds to reference probe molecules in a cell line or a panel of cell lines.

Label-free techniques are powerful for lead and probe identification, in particular for poorly characterized targets. Using chemical similarity search based on hits, fragments or substructures identified from the HTS campaign, one can quickly expand compound library and follow-up with label-free profiling to discover potent probes, and even endogenous agonist(s), for the novel target [14]. Label-free techniques can also be used to assess the safety of lead-like molecules. First, almost all drugs display polypharmacology, the ability to bind to more than one target. Polypharmacology could be beneficial or harmful, depending on the targets bound by the drug. RWG has high sensitivity to detect functional receptome in living cells ranging from $G$ protein-coupled receptors to receptor tyrosine kinases, ion channels, transporter, immune receptors, cytoskeletal proteins and kinases, enabling multitarget profiling [6,13,14]. Label-free profiling with an enlarged panel of cell lines can dramatically increase the target coverage for polypharmacology assessment of drug molecules. MS can assess polypharmacology of drugs acting at the entire human proteome, thus facilitating the identification of primary target(s) and toxicityinducing anti-target(s) for the drugs [16]. Second, druginduced toxicities in liver, heart, kidney and brain currently account for more than $70 \%$ of drug attrition and 
drug withdrawal. The toxicity potential of drug candidates prior to in vivo studies can be assessed in vitro using highly predictive cellular models. Label-free has been used to assess compound-induced hepatotoxicity, neurotoxicity and cardiotoxicity $[6,17,18]$. Third, labelfree profiling with distinct probe- and gene-modulated cells provides a high-resolution self-referenced means to differentiate and classify lead-like molecules into different clusters, so leading candidates can be selected for in vivo testing $[19,20]$. Fourth, combining label-free profiling in disease-relevant cell models with text mining, one can predict the biological functions of novel targets. For instance, based on the discovery of cromolyn disodium and nedocromil sodium, the two clinically used antiasthma chromones, as well as certain natural phenols as GPR35 agonists, we hypothesized and confirmed that several phytochemicals including gallic acid and wedelolactone, known to suppress allergic reaction-related responses, are also GPR35 agonists [21].

Label-free imaging techniques are useful for chemical and phenotypic profiling of living cells [22]. MS can be used to map out drug metabolites in living cells. In-cell NMR can provide novel insights into structural biology and drug-target interactions in the crowded native cellular environments. Nonlinear optical microscopies (e.g., two-photon fluorescence, second-harmonic generation and digital holographic microscopy) enable chemical and morphological mapping of living cells and tissues. Coherent anti-Stokes Raman scattering and stimulated Raman scattering

\section{References}

1 Pacholec M, Ble casdale JE, Chrunyk B et al. SRT1720, SRT2183, SRT1460, and resveratrol are not direct activators of SIRT1. J. Biol. Chem. 285(11), 8340-8351 (2010).

$2 \mathrm{Hu} \mathrm{H}$, Deng H, Fang Y. Label-free phenotypic profiling identified D-Luciferin as a GPR35 agonist. PLoS ONE 7, e34934 (2012).

3 Rask-Andersen M, Almén MS, Schiöth HB. Trends in the exploitation of novel drug targets. Nat. Rev. Drug Discov. 10(8), 579-590 (2011).

4 Dar AC, Das TK, Shokat KM, Cagan RL. Chemical genetic discovery of targets and anti-targets for cancer polypharmacology. Nature 486(7401), 80-84 (2012).

5 Swinney DC, Anthony J. How were new medicines discovered? Nat. Rev. Drug Discov. 10(7), 507-519 (2011).

$6 \quad$ Fang Y. Label-Free Biosensor Methods In Drug Discovery. Springer, New York, NY, USA (2015).

7 Fang Y. Ligand-receptor interaction platforms and their applications for drug discovery. Exp. Opin. Drug Discov. 7(10), 969-988 (2012).

8 Copeland RA, Pompliano DL, Meek TD. Drug-target residence time and its implications for lead optimization. Nat. Rev. Drug Discov. 5(9), 730-739 (2006). microscopies permit imaging specific molecules, macromolecular assembles and organelles of living cells. Together, it is possible to probe many differ aspects of drug pharmacology in living cells and tissues that are otherwise difficult to be studied by conventional labeling techniques.

In summary, label-free investigations are well positioned to facilitate early drug discovery. For followon drug discovery where the molecular mechanism of action is known, label-free can be implemented in a way similar to conventional label-dependent screens, and lead-like molecules can be optimized and advanced based on binding affinity, kinetics, and efficacy. For unproven targets where the desired molecular mechanism of action is unknown, multiple label-free and dependent techniques shall be combined to provide systematic comprehension of the druggability of the target and lead-like molecules.

\section{Financial \& competing interests disclosure}

The author is a Research Director/Fellow and stockholder of Corning Incorporated. Epic ${ }^{\circledR}$ systems and Dynamic Mass Redistribution assays that employ RWG in microplate are commercial products of Corning Incorporated. The author has no other relevant affiliations or financial involvement with any organization or entity with a financial interest in or financial conflict with the subject matter or materials discussed in the manuscript apart from those disclosed.

No writing assistance was utilized in the production of this manuscript.

9 Goral V, Jin Y, Sun H, Ferrie AM, Wu Q, Fang Y. Agonistdirected desensitization of the $\beta_{2}$-adrenergic receptor. PLoS ONE 6, e19282 (2011).

10 Deng H, Wang C, Su M, Fang Y. Probing biochemical mechanisms of action of muscarinic $\mathrm{M}_{3}$ receptor antagonists with label-free whole-cell assays. Anal. Chem. 84(19), 8232-8239 (2012).

11 Yuan Y, Pei J, Lai L. Binding site detection and druggability prediction of protein targets for structurebased drug design. Curr. Pharm. Des. 19(12), 2326-2333 (2013).

12 Pellecchia M, Bertini I, Cowburn D et al. Perspectives on NMR in drug discovery: a technique comes of age. Nat. Rev. Drug Discov. 7(9), 738-745 (2008).

13 Fang Y. Label-free drug discovery. Front. Pharmacol. 5, 52 (2014).

14 Fang Y. Combining label-free cell phenotypic profiling with computational approaches for novel drug discovery. Exp. Opin. Drug Discov. 10(4), 331-343 (2015).

15 Dodgson K, Gedge L, Murray DC et al. A 100K well screen for a muscarinic receptor using the Epic label-free system - a reflection on the benefits of the label-free approach to screening seven-transmembrane receptors. J. Recept. Signal Transduct. Res. 29(3-4), 163-172 (2009). 
16 Savitski MW, Reinhard FB, Franken $\mathrm{H}$ et al. Tracking cancer drugs in living cells by thermal profiling of the proteome. Science 346, 1255784 (2014).

17 Abassi YA, Xi B, Li N et al. Dynamic monitoring of beating periodicity of stem cell-derived cardiomyocytes as a predictive tool for preclinical safety assessment. $\mathrm{Br}$. J. Pharmacol. 165(5), 1424-1441 (2012).

18 Ferrie AM, Wu Q, Deichmann O, Fang Y. High frequency resonant waveguide grating imager for assessing druginduced cardiotoxicity. Appl. Phys. Lett. 104, 183702 (2014).
19 Ferrie AM, Sun H, Fang Y. Label-free integrative pharmacology on-target of drugs at the $\beta_{2}$-adrenergic receptor. Sci. Rep. 1, 33 (2011).

20 Ferrie AM, Sun H, Zaytseva N, Fang Y. Divergent label-free cell phenotypic pharmacology of ligands at the overexpressed $\beta_{2}$-adrenergic receptors. Sci. Rep. 4, 3828 (2014).

21 Deng H, Fang Y. Anti-inflammatory gallic acid and wedelolactone are $\mathrm{G}$ protein-coupled receptor-35 agonists. Pharmacology 89(3-4), 211-219 (2012).

22 Fang Y. Label-free chemical and phenotypic profiling of living cells. Science Letters 4, 156 (2015). 